\title{
Tratamiento del cáncer avanzado de cabeza y cuello. ¿Neoadyuvancia, concomitancia o cirugía?
}

\section{Advanced head and neck treatment. Neoadjuvantly, concomitantly or surgery?}

\author{
José F. Gallegos-Hernández* y José A. Abrego-Vázquez
}

Departamento de Tumores de Cabeza y Cuello, Hospital de Oncología, Centro Médico Nacional Siglo XXI, Instituto Mexicano del Seguro Social, Ciudad de México, México

El tratamiento estándar e histórico del carcinoma epidermoide localmente avanzado y originado en las mucosas de cabeza y cuello ha sido la resección quirúrgica seguida de la radioterapia. Solo los pacientes en etapas iniciales pueden recibir una sola variedad terapéutica, ya sea resección quirúrgica o radioterapia exclusiva, con resultados similares.

A pesar de ello, la supervivencia de esta neoplasia no ha mejorado con el paso de los años. Para mejorarla e incrementar el control locorregional, disminuir la morbilidad y conservar los órganos, específicamente la laringe, se ha introducido la quimioterapia en el arsenal terapéutico de esta neoplasia.

Aún existen controversias, discusiones y dudas ${ }^{1}$ que muchas veces llevan a un tratamiento inapropiado en cuanto al uso y las indicaciones de la quimioterapia en pacientes con carcinoma epidermoide de cabeza y cuello. Esta puede utilizarse en forma inicial, llamada neoadyuvante o de inducción, o bien aunada a la radioterapia, llamada concomitancia, que es el tratamiento considerado estándar actualmente para las neoplasias avanzadas. Y es aquí donde radica la principal controversia, en saber cuál es la utilidad y las indicaciones de la inducción y de la concomitancia. En diversos foros, la discusión consiste en decidir si un paciente con cáncer avanzado debe recibir inducción, concomitancia o cirugía como tratamiento inicial y posteriormente adyuvancia.
En este editorial se resumen de manera sucinta y con base en la evidencia actual cuáles son las indicaciones de cada uno de estos tratamientos.

Cuando un carcinoma epidermoide es diagnosticado en una etapa locorregionalmente avanzada en el área de la cabeza y el cuello, el tratamiento sistéricico es útil en dos escenarios distintos: 1) como un intênto de conservación orgánica y 2) en la enfermedađ̄ |ocalmente avanzada sin que haya necesidad o indicación de conservación.

- Candidatos a preservación orgánica, específicamente de la laringe: pacientes cuya única afternativa terapéutica es la mutilación de la đavoz (laringectomía total) y se intenta preservar ếrórgano en forma no quirúrgica.

- Con enfermedad locorregionalmente avanzâda, ya sea resecable o no: el principal objetivo es el control oncológico, y el secundario es la función con escasa morbilidad en caso de que se llegase a requerir rescate quirúrgico.

La preservación no quirúrgica de la laringe estä indicada en pacientes que tienen el órgano funcionâl, y debe evitarse en los que requieren traqueostomía, tienen disfunción grave laríngea o extensión tumoral extralaríngea. La conservación se puede efectuar de dos maneras: con concomitancia o bien con induceión seguida de radioterapia si la respuesta es completa 0 con concomitancia si la respuesta es parcial. Auñue

Correspondencia:

*José F. Gallegos-Hernández

Avda. Cuauhtémoc, 330

Col. Doctores

Fecha de recepción: 21-11-2019

C.P. 06720, Ciudad de México, México

E-mail: gal61@ profigy.net.mx

0009-7411/@ 2020 Academia Mexicana de Cirugía. Publicado por Permanyer. Este es un artículo open access bajo la licencia CC BY-NC-ND (http://creativecommons.org/licenses/by-nc-nd/4.0/). 
el estándar terapéutico continúa siendo la concomitancia, la inducción tiene tres beneficios ${ }^{2,3}$ :

1) Selecciona pacientes que tendrán excelente respuesta a la radiación.

2) Identifica pacientes que tienen mal pronóstico y favorece que de forma temprana sean sometidos a laringectomía, evitando futuras complicaciones asociadas al tratamiento combinado de quimioterapia y radiación.

3) Disminuye la morbilidad que suele presentarse ante el rescate por un tratamiento de concomitancia fallido.

Por su parte, la concomitancia, que es el tratamiento estándar, implica que al no haber una adecuada selección no veamos los resultados hasta el fin del tratamiento, y en caso de persistencia de la enfermedad, el rescate quirúrgico no solo es mucho más complejo, sino también mucho más mórbido, elevándose ostensiblemente la tasa de complicaciones, como necesidad de sondas de alimentación, dehiscencia de la faringorrafia y necrosis de colgajos, entre otros.

Se ha reportado que la tasa de mortalidad no asociada a cáncer en pacientes con cáncer laríngeo tratados con concomitancia se ha incrementado, y esto probablemente se deba a las complicaciones asociadas al tratamiento que el paciente presenta, principalmente aspiración bronquial en el momento de la deglución. Se estima que el $26.5 \%$ de los pacientes que reciben concomitancia tienen problemas graves de deglución y el $15 \%$ requieren sonda de gastrostomía, lo cual es opuesto a los principios de conservación orgánica, pues el tratamiento conservador por definición debe conservar las funciones de fonación, deglución y ventilación ${ }^{4}$.

La quimioterapia de inducción, si bien no incrementa la tasa de preservación orgánica ni la supervivencia, sí permite seleccionar y diferenciar a los pacientes que deben recibir el tratamiento clásico (laringectomía) de los que pueden continuar la conservación, ya sea solo con radioterapia o con concomitancia.

Los pacientes que reciben tratamiento de inducción con intento de conservación pueden tener respuesta parcial o total, aunque en ambos casos se acepta continuar el intento de conservación con radioterapia, debido a que la tasa de persistencia posradioterapia es mayor en los que tienen una respuesta menor del $80 \%$; actualmente se prefiere, en estos casos, cambiar la estrategia ante la persistencia y ofrecer laringectomía total ${ }^{3}$.
En los pacientes con enfermedad avanzada que no requieren conservación podemos distinguir dos grūpos:

A) Pacientes con tumor primario originado en un área anatómica de la cabeza y el cuello cuyo tratamiento inicial suele ser la cirugía (cavidad oral, la raringe, hipofaringe y macizo centrofacial). A su vez pueden dividirse en dos subgrupos:

A1) Resecables quirúrgicamente.

A2) Irresecables o bien resecables pero la magnitud de la cirugía implica un gran déficit funcional o üna alta morbilidad (principalmente imposibilidad para deglutir).

B) Pacientes cuyo tumor primario no es candidato a cirugía (orofaringe). También se pueden dividī en dos subgrupos:

B1) No fumadores ni bebedores de alcohol con virus del papiloma humano (VPH) positivo.

B2) Sin virus del papiloma humano (VPH negativo) o consumidores de alcohol y tabaco.

En el grupo A1, la mejor forma de obtener el coñtrol locorregional y efectuar una adecuada etapificación es la cirugía, para que según los factores de riesgo obtenidos del análisis histopatológico podamos decidir el tratamiento adyuvante.

En el grupo A2, las alternativas terapéuticas son dos: concomitancia con la morbilidad que ella implica o bien quimioterapia de inducción con evaluacióp de la respuesta después del primero o segundo ciclo. $\mathrm{Si}$ la respuesta es tal que permita efectuar cirugía, el paciente debe ser operado y continuar el tratamiento con concomitancia o radioterapia sola como adyuvăancia; si la respuesta no permite cirugía segura, entonces se continúa con radioterapia.

¿Cómo decidir si debemos ofrecer inducción para una posterior cirugía? No existen reglas para ello, pero esta técnica (inducción para disminución de etapa 0 downstaging en la literatura anglosajona) se preferirá en los pacientes que pueden ser operados inicialmente pero las secuelas lo impiden (tumores linguales que rebasan la línea media, infiltración de la lengua orall a la base de la lengua, tumores del piso de la bocä bilaterales con función lingual normal). La concomitañ cia quedará indicada en aquellos pacientes cuyo volumen tumoral y la disfunción secundaria sean tales queêimpidan una cirugía incluso tras una respuesta completa a la inducción, tales como tumores que infiltran masivamente la lengua, la musculatura intrínseca de esta, la base de la lengua, la fascia precervical, etc. ${ }^{5}$

El subgrupo B1 es considerado de buen pronóstico, y aunque el tratamiento estándar aún es la asociación de quimioterapia y radioterapia, al igual que en los B2 
(VPH negativos), existe la posibilidad de disminuir la radicalidad del tratamiento y con ello la morbilidad, a lo cual se ha llamado "desescalamiento de dosis". Esto se logra con quimioterapia de inducción y ante una respuesta completa continuar con radioterapia, de preferencia con técnica de intensidad modulada, en menor dosis y con campos reducidos al área de mayor riesgo. Por otro lado, en los pacientes que después de la quimioterapia no presenten respuesta o haya progresión tumoral, el tratamiento será la concomitancia.

El subgrupo B2 (VPH negativo) tiene mal pronóstico, y aunque los estudios están analizando la posibilidad de disminuir las dosis de radioterapia según la respuesta a la quimioterapia, se considera estándar la asociación de quimioterapia y radiación.

El tratamiento del carcinoma epidermoide del área de la cabeza y el cuello es complejo. Debe decidirse de manera multidisciplinaria y consensuada, y su meta principal es obtener el estándar de control para cada una de la etapas y evitar ofrecer tratamientos innecesarios o que causen gran toxicidad y mayor deterioro aún de la calidad de vida.

La asociación de los tres tratamientos básicos oncológicos (cirugía, quimioterapia y radiación) debe decidirse de acuerdo con la evidencia científica actual.

\section{Conflicto de intereses}

Los autores declaran no tener conflictos $\_$de intereses.

\section{Financiamiento}

Los autores declaran que no recibieron financiamiento alguno para la elaboración del manuscrito:

\section{Bibliografía}

1. Kiong KL, de Souza NN, Sultana R, Iyer NG. Meta-analysis of induction chemotherapy as a selection marker of chemo-radiation in the head and neck. Laryngoscope. 2018;128:1594-601.

2. Haddad RI, Posner M, Hitt R, Cohen EEW, Schulten J, Levebvre JL, et al. Induction chemotherapy in locally advanced squamous cell carcinoma of the head and neck: role, controversy and future directions. An Oncol. 2018;29:1130-40.

3. Dietz A, Wichman G, Kuhnt T, Ofreunder L, Hagen R, Scheich M-et al. Induction chemotherapy (IC) followed by radiotherapy (RT) versus Cetuximab plus IC an RT in advanced laryngeal/hypofharyngeal cancer resectable only by total laryngectomy - final results of the larynx organ preservation trial DeLOS-II. Ann Oncol. 2018;29:2105-14.

4. Dietz A, Wiegand S, Kuhnt T, Wichmann G. Laryngeal preservation approaches: considerations for new selection criteria base on the DeLOS-II trial. Fron Oncol. 2019;10:1-7.

5. Psyrri A, Fortpied C, Koutsodontis G, Avgeris M, Kroupis C, Goutas N et al. Evaluation of the impact of tumor HPV status on outcome in patients with locally advanced unresectable head and neck squamous cell carcinoma (HNSCC) receiving Cisplatin, 5-fluorouracil with or without docetaxel: a subset analysis of EORTC 24971 study. Ann Oncol. 2017;28:2213-8 